befördert, weil durch diese die Kohlensäure ausgetrieben und die Berührung mit der Luft vermehrt wird, deren Sauerstoff das Eisenoxydul höher oxydirt. Von den Erwärmungsmethoden sind die mit heissem Wasser (C) und mit directer Feuerung (D) weniger vortheilhaft, weil dieselben die Zersetzung des Wassers befördern; dagegen duirfte die Dampfheizung (E) am vortheilhaftesten sein, indem hier das Wasser am wenigsten verändert wird, und die Dampfheizung nach der Schwartz'schen Methode, wenn die Dampfapparate einmal vorhanden sind, auch leicht eingerichtet werden kann, wenn diese sich als noch zweckmässiger bewähren sollte.

Die Resultate vorliegender Versuche sind noch mehr in die Augen fallend, wenn man den procentischen Unterschied der Niederschläge, von denen doch in der frühern aufgelösten Form die Wirksamkeit des Mineralwassers abhängig ist, betrachtet. Der Niederschlag

sub C. betrug 0,024 Grm.,

sub D. " $0,045 \%=33 \frac{1}{3}$ Proc. und

sub E. " 0,007 " $=66 \frac{2}{3}$ " Unterschied; d. h. die letztere Erwärmungsmethode ist um $\frac{1}{3}$ und $\frac{2}{3}$ besser, als die vorhergehenden. Das auf die letzte Art er wärmte Wasser behält so lange Zeit, als zum Baden erforderlich ist, alle seine Bestandtheile in Auflösung, da es fast nicht schneller, als das nicht erwärmte und der Ruhe überlassene Mineralwasser zersetzt wird.

\title{
Bereitung des Borax (zweifach borsauren Natrons); \\ von
}

O. Koehnke.

Obgleich die chemischen Fabriken viele pharmacentisch-chemische Präparate zu sehr billigen Preisen liefern, die der Apotheker nicht im Stande ist dafür herzustellen, so sind doch noch manche, und ich möchte glauben, die meisten chemischen Präparate, besonders in grossen Apo- 
theken, mit lohnendem Vortheil selbst zu bereiten, wenn man, wie dieses in Fabriken geschieht, von den Landespharmakopöen abweichende Bereitungsmethoden befolgt. Beispielsweise erlaube ich mir nur einige Präparate anzuführen: Acetum concentratum, bereitet durch Zerlegung des essigsauren Kalks, und dieser dargestell aus gutem Aetzkalk und rohem durch die Schnellessigfabrication gewonnenem Essig; Acidum boracicum, durch Reinigung der rohen toskanischen Borsäure mit Berücksichtigung der Vorschrift von Wack enroder; Aether aceticus, aus essigsaurem Kalk, anstatt des Bleizuckers, essigsauren Kalis oder Natrons; Alcohol absolutum, durch Behandlung des Weingeistes mit roher Pottasche und Aetzkalk, nach Soubeiran's Angabe; Kali aceticum und Acidum tartaricum, durch Zersetzung des neutralen weinsteinsauren Kalis mit essigsaurem Kalk, und ferner durch Zerlegung des schwefelsauren Kalis vermittelst essigsauren Kalks. Ueberhaupt lassen sich fast alle in der Pharmacie gebräuchlichen essigsauren Salze vermittelst eines schwefelsauren Salzes und Zerlegung desselben mit essigsaurem Kalk billig und sehr rein darstellen. Kali chloricum, bereitet nach Liebig's, Liquor Ammonii caustici nach Wackenroder's, Spiritus nitriconethereus, durch Behandlung von Stärke mit Salpetersäure nach Liebig's Vorschrift. Sulfur praecipitatum, dargestellt durch Fällung einer Schwefelcalciumlösung; Tartarus stibiatus, nach der Vorschrift von Wittstock; Zincum oxydatum, nach Wackenroder's oder Dufl os' Methode u. s. w. Hierher gehört denn auch der von Fabriken vorschriftsmässig zu beziehende Borax. Soll bei diesem Präparate indess ein lohnender pecuniairer Vortheil erzielt werden, so ist es nothwendig, mit grössern Mengen, etwa wie in nachstehender Angabe folgt, zu arbeiten.

Man verwendet entweder rohes krystallisirtes kohlensaures Natron und rohe toskanische Borsäure, oder anstatl des ersteren entweder Alikantische, auch Teneriffa-Soda, oder auch drittens ostindischen halbraffinirlen Borax.

Bei der Darstellung des Borax aus rohem krystallisirtem kohlensaurem Natron und roher toskanischer Bor- 
säure bereitet man sich eine kaustische Natronlauge von ungefähr 170 Pfund, deren spec. Gew. 1,090-1,095 ist, wozu durchschnittlich 50 Pfund Natron und 30 Pfund guter Aetzkalk, letzterer mit dem vierfachen Gewichte Wassers zu einem Kalkbrei gebracht, erforderlich sind.

Nachdem die Mischung in einem eisernen Kessel nach den Regeln der Kunst gekocht und in eine Aetznatronlauge verwandelt ist, bedeckt man denselben sorgfältig und zieht nach einigen Stunden die klare Lauge vermittelst eines Hebers ab, übergiesst den Rückstand mit einer angemessenen Menge Wassers, rührt gut um und zieht abermals nach einigen Stunden die klare Flüssigkeit ab. Der Rückstand wird nun zum zweiten Male mit Wasser hingestellt und später abgegossen, worauf diese höchst verdünnte Lauge zu den Abwaschungen der in der Folge erhaltenen Boraxkrystalle verwandt wird.

Die also erhaltenen Laugen werden zu 170 Pfund oder dem spec Gew. von 1,090-4,095 eingekocht und nunmehr nach und nach $40 \mathrm{Pfd}$. gute toskanische Borsäure eingetragen und weiter gekocht, bis die Lauge $120-125$ Pfd. beträgl, oder das spec. Gew. von 1,175-1,180 zeigt, welches den von Berzelius angegebenen $20-22^{\circ}$ Baumé entspricht. Hierauf wird die Flissigkeit siedendheiss in einen hölzernen Bottich gegossen, der mit wollenen Decken und Stroh gut umwickelt ist und sorgfältig bedeckt wird, um die Wärme so lange wie möglich zu erhalten, wodurch nur eine gute und regelmässige Krystallisation bewirkt werden kann.

Krystallisirt der Borax in einer Wärme, die 35 bis $4.0^{\circ}$ beträgt, so erhält man bekanntlich oktaedrische Krystalle, die nur 5 At. Krystallwasser enthalten und desshalb einen bedeutenden Verlust an Ausbeute herbeiführen. Dieses ist jedoch nicht bei obigem Verfahren zu befürchten. Dagegen hat man aber hinsichtlich des spec. Gew. der Lauge eine sehr grosse Aufmerksamkeit zu verwenden; denn wird die zum Krystallisiren hingestellte heisse Lauge weiter eingekocht, als zu dem spec. Gew. von 1,180, so bekommt man ebenfalls oktaedrische Krystalle. Ausserdem 
ist zu bemerken, dass die Borsäure, welche das erste Mal nach obiger Angabe hinzugesetzt worden ist, lange nicht hinreicht, um alles Natron in zweifach borsaures zu verwandeln, das Natron aber in bedeutend vorherrschender Menge eine vortheilhafte Krystallisation bewirkt. Dieses möchte darin seinen Grund finden, dass viele Salze mit einem gewissen Ueberschuss an Basis besser und regelmässiger krystallisiren. Ferner ist es vortheilhaft, die Aetznatronlauge nicht von dem aufgelösten kohlensauren (?) Kalk zu befreien, sondern lieber noch etwas freien Aetzkalk hinzuzusetzen, da diese zur theilweisen Zersetzung der schwefelsauren Verbindungen der rohen Borsäure dienen.

Nach drei Tagen ist die erste Krystallisation beendigt. Die gesammelten Krystalle werden zerschlagen, mit der früher gedachten und zurückgesetzten verdünnten Lauge gut abgewaschen und zurückgestellt, hingegen die abgegossene Boraxlauge mit den Abwaschflüssigkeiten zum Absetzenlassen des sich hauptsächlich gebildeten schwefelsauren Kalks und der sonstigen mechanisch hineingebrachten Unreinigkeiten hingestellt. Sobald die Lauge sich abgesetzt hat, wird sie vorsichtig klar abgezogen, eingekocht und während dessen abermals 8 Pfd. Borsäure allmälig hinzugesetzt und wie vorher behandelt. Die nunmehr zurückbleibende Lauge erfordert noch 2 bis 5 Pfd. Borsäure, was man durch eine vorläufige Probe leicht findet, jedoch darf nicht übersehen werden, dass, wie bereits gedacht, das Natron vorwalte oder vielmehr blaues Lackmuspapier nicht geröthet werde, worauf nach dieser dritten Krystallisation die zurückbleibende Lauge, mit Schwefelsäure gesättigt, noch auf etwas schwefelsaures Natron benutzt werden kann.

Die Umkrystallisation der sämmtlich erhaltenen Krystalle muss meistens zweimal wiederholt werden, zu welchem Zwecke man selbige in $2 \frac{1}{2}$ Theilen Regenwasser durch Kochen auflöst, die Lauge zu dom crwähnten spec. Gew. bringt und siedendheiss in ein hölzernes, vor jeder raschen Abkühlung geschützles Gefäss bringt. Die Eindampfung 
der rückständigen Lauge wird wiederholt. Binnen 2 bis 3 Tagen ist die Krystallisation immer beendigt. Jede Filtration, ausser bei der letzten Umkrystallisation, ist überfluissig.

Die Ausbeute beträgt, wenn man recht gute Substanzen angewandt hat, bei umsichtiger Behandlung 60 bis 62 Pfd. an reinem krystallisirtem Borax.

Vortheilhafter, indess schwieriger, ist die Bereitung des Borax aus roher Soda und Borsäure. Zu diesem Zwerk stellt man sich ebenfalls eine Aetznatronlauge dar, die 300 Pfd. beträgt, deren spec. Gew. 1,090-1,095 ist, wozu ungefähr $100 \mathrm{Pfd}$. gute rohe Alikantische Soda, bekannt unter dem Namen Barilla-, oder besser Teneriffa-Soda, und 45 bis 50 Pfd. Aetzkalk erforderlich sind. Die Lauge wird auf dieselbe Weise, wie vorher, dargestellt und alsdann 45 bis $48 \mathrm{Pfd}$. toskanische Borsäure nach und nach eingetragen, worauf die Lauge zu ungefähr 180 bis $185 \mathrm{Pfd}$. oder zu dem spec. Gew. von 1,175-1,180 eingekocht, unterdessen von Zeit zu Zeit abgeschäumt und nun zum Krystallisiren hingestellt wird. Zu der ersten Multerlauge setzt man alsdann 8 bis 10 Pfd. Borsäure, und zu der zweiten rückständigen Lauge noch 2 bis 3 Pfd., oft aber auch, je nach den angewandten Substanzen, bis $10 \mathrm{Pfd}$. Borsäure, worüber vorherige Untersuchungen mit der Boraxlauge entscheiden müssen. Die Bearbeitung ist uibrigens die nämliche, welche bei der vorigen Darstellung des Borax angegeben ist. Aus der letzten Mutterlauge, mit Schwefelsäure gesättigt, erhält man übrigens eine grössere Menge schwefelsaures Natron.

Die Ausbeute von krystallisirtem Borax beträgt 80 bis 90 Pfund.

Die dritte Bereitungsweise des zweifach borsauren Natrons, die wohl schwerlich in pharmaceutischen Laboratorien, vielleicht aber versuchsweise, Nachahmung finden möchte, da bei der Bearbeitung manche empirischeKenntnisse erfordert werden, erlaube ich mir nur kurz anzudeuten. Hierzu wird am zweckmässigsten der ostindische halbraffinirte Borax, welcher sehr viele schmierige oder fettige 
Bestandtheile enthält, mit einer Aetznatronlauge von 1,370 bis 1,380 spec. Gew. gewaschen, alsdann, da er immer einen bedeutenden Ueberschuss an Natron enthält, mit toskanischer Borsäure nach und nach, wie bei den frühern Bearbeitungen der Boraxlaugen angegeben worden, gesältigt, und durch 3 - bis kmaliges Umkrystallisiren mit alle den bereits erwähnten und mehreren Vorsichtsmaassregeln gereinigt. Die Ausbeute kann 63 , und im günstigsten Falle bis zu 75 Proc. an krystallisirtem Borax betragen.

Nach dieser Zusammenstellung von Methoden, die in der Praxis wiederholt ausgefuihrt wurden, wird ein Vergleich mit den Preiscouranten nicht schwer und daraus ein in pecuniairer Hinsicht recht güstiges Resultat ersichtlich sein.

\section{Bereitung des Kali carbon. acidulum; von Veling, Apotheker in Hillesheim.}

Schon seit mehren Jahren bediene ich mich bei Bereitung dieses Salzes eingemengter Kohle.

Ich verfahre dabei wie folgt. Die möglichst von fremden Salzen gereinigte Pottaschenlauge verdunste ich so weit, bis die Masse anfängt sich zu ballen; nun setze ich ihr ein ungefähr gleiches Volumen Kohlenpulver zu und verarbeite es zu einer gleichförmigen Masse, der ich, wenn sie schon zu trocken hierzu sein sollte, so viel Wasser zusetze, um diess möglich zu machen, erwärme so lange, bis die warme Masse noch feucht erscheint, oder sich noch klümpert. Nun fülle ich sie in Tüten von $\frac{1}{4}$ Bogen Fliesspapier und lege solche im Wohnzimmer an einen möglichst hohen, nicht feuchten Ort und iiberlasse sie sich selbst während 12 bis 15 Wochen. Man wird sie nun in Kali bicarbon. umgeändert finden.

Diese Methode lässt sich aber nur im Winter in einer geheizten Stube ausfuhren, wo die Luft möglichst gleich- 